\title{
Mutations des ressources clientélaires et construction des notabilités politiques à Marseille (1970-1990)
}

In: Politix. Vol. 17, N67. Troisième trimestre 2004. pp. 129-155.

\begin{abstract}
Résumé
Mutations des ressources clientélaires et construction des notabilités politiques à Marseille (1970-1990)

Cesare Mattina

A Marseille, la distribution des ressources publiques, en particulier des emplois municipaux et des logements sociaux entre les années 1960 et 1980, à travers des canaux familiaux et clientélaires, a représenté un véritable facteur de régulation politique et sociale de la ville. Cependant le rétrécissement de ces ressources dans les deux dernières décennies a fortement remis en question les mécanismes de régulation sociale à travers la redistribution clientélaire des ressources. Les processus et les parcours de notabilisation des hommes politiques en ont été par conséquent affectés. L'incapacité (ou la moindre efficacité) à répondre aux demandes incessantes des électeurs en termes de services et de faveurs personnels a fini par amoindrir le prestige notabiliaire. Le rôle et la fonction de médiation symbolique du notable ont donc pris une plus grande importance.
\end{abstract}

\section{Abstract}

he Tranformation of Clientelistic Resources and the Construction of Political Notability in Marseille (1970-1990)

\section{Cesare Mattina}

In Marseille, the clientelistic distribution of public employments and social housing between 1960 and 1980 represented a factor of political and social regulation in the city. However the decreasing of those traditional public resources in two last decades strongly weakened this kind of regulation. One of the consequences is the change in the characteristics of elected representatives' notability. The difficulties to answer to the electors who ask preferential treatments have reduced the prestige of notability. Notables' function and rule are progressively transformed into those of "symbolic broker".

Citer ce document / Cite this document :

Mattina Cesare. Mutations des ressources clientélaires et construction des notabilités politiques à Marseille (1970-1990). In: Politix. Vol. 17, N67. Troisième trimestre 2004. pp. 129-155.

doi : 10.3406/polix.2004.1627

http://www.persee.fr/web/revues/home/prescript/article/polix_0295-2319_2004_num_17_67_1627 


\section{Mutations des ressources clientélaires et construction des notabilités politiques à Marseille (1970-1990)}

Cesare MatTina

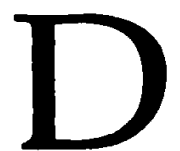

ans une grande partie de l'historiographie française, le notable a été défini par son héritage familial et par sa position sociale à l'intérieur d'une société locale. La domination politique du notable découlait ainsi directement de sa domination sociale et économique sur un territoire (le plus souvent un village ou une petite ville), l'élection ne faisant que confirmer et légitimer cette domination ${ }^{1}$. D'un autre côté, la sociologie des organisations a proposé une définition fonctionnelle du notable, considéré comme le point de jonction entre la population locale et les agents de l'Etat central ${ }^{2}$. Le notable a été envisagé dans ce cas en fonction de ses capacités de tisser des relations avec les services étatiques en vue de la défense des intérêts locaux ${ }^{3}$. En insistant sur la position sociale du notable et sur sa fonction de relais "vertical " entre bureaucratie étatique et population, ces types d'analyse ont laissé dans l'ombre deux éléments

1. Tudesq (A.), «Le concept de "notable" et les différentes dimensions de l'étude des notables », Cahiers de la Méditerranée, 47, 1993 ; Charle (C.), "Légitimité en péril. Eléments pour une histoire comparée des élites et de l'Etat en France et en Europe occidentale (XIXe-XXe siècles) ", Actes de la recherche en sciences sociales, 116-117, 1997.

2. Grémion (P.), Le pouvoir périphérique. Bureaucrates et notables dans le système politique français, Paris, Le Seuil, 1976.

3. Worms (J.-P.), « Le préfet et ses notables ", Sociologie du travail, 3, 1966. 
influant lourdement sur les processus de notabilisation : les pratiques liées à l'exercice du métier d'élu et l'inscription du notable dans des réseaux relationnels, dans des environnements de contraintes et d'opportunités, au sein d'une société localisée. Notre questionnement porte ici sur ces éléments. Il ne s'agit pas tellement de s'interroger sur les raisons qui font qu'un homme politique est ou devient un notable, mais plutôt sur la manière dont la notabilité est alimentée et entretenue à travers l'exercice du métier politique dans des territoires précis (quartiers, cités, circonscriptions, secteurs urbains, etc.) ainsi que sur les modalités à travers lesquelles la demande de services et de faveurs personnelles, adressée sans cesse par la population vers les élus, influe sur l'exercice du métier politique et sur les processus de la notabilisation. Les études historiques et la sociologie des organisations apparaissent en outre plus adaptées à des contextes ruraux qu'à des milieux urbains. En revanche, dans des grandes villes telles que celles que nous avons étudiées 5 , les stratégies et les parcours notabiliaires s'insèrent souvent dans des structures partisanes et dans des machines politiques municipales. Les ressources notabiliaires n'apparaissent pas uniquement liées au prestige social, à la richesse économique et à la capacité de nouer des relations avec les services de l'Etat. Elles dépendent aussi de la capacité de s'assurer une carrière dans un parti et de la maîtrise, à l'intérieur des collectivités locales, des leviers de la redistribution des biens publics (emplois, logements sociaux, différentes allocations et subventions).

Afin d'analyser le processus de construction et d'entretien de la notabilité, nous avons pris comme point d'observation privilégié un des aspects qui est considéré comme typique de l'activité du notable : les pratiques d'échange en relation avec la construction d'une clientèle politique. Notre questionnement porte donc sur la façon dont les échanges clientélaires de ressources à l'intérieur de divers cercles sociaux (groupe familial, cercles d'amis ou de militants, électeurs, etc.) participent à la construction d'une notabilité politique ainsi que sur la façon dont l'inscription des élus dans un parti et leurs fonctions dans les institutions s'articulent avec les ressources sociales (notoriété, prestige, etc.) qu'ils ont accumulées dans le cours de leur carrière et qu'ils peuvent faire valoir dans les territoires au sein desquels ils agissent. Notre étude a comme terrain la ville de Marseille dans la période

4. A propos de l'œuvre de Grémion, il a été par exemple remarqué que son "schéma ne s'applique pas aux députés-maires, ni aux grandes villes, ni aux municipalités communistes ; qu'il ne tient pas compte du pouvoir économique; que son interprétation aussi convient essentiellement à un espace départemental à dominante rurale " (cf. Mabileau (A.), "Les génies invisibles du local. Faux-semblants et dynamiques de la décentralisation ", Revue française de science politique, 47 (3-4), 1997, p. 347).

5. Mattina (C.), La régulation clientélaire. Relations de clientèle et gouvernement urbain à Naples et à Marseille (1970-1980), Thèse pour le doctorat de science politique, IEP de Grenoble, 2003. 
comprise entre les années 1970 et les années 1990. Dans cette ville, la distribution des ressources publiques (en particulier des emplois et des logements sociaux) à travers des canaux familiaux et clientélaires a représenté un véritable facteur de régulation politique et sociale à partir des années $1960^{6}$. La distribution prioritaire à certains individus et groupes sociaux ou ethniques de ces biens a été un facteur souvent déterminant $\mathrm{d}^{\prime}$ ascension et $\mathrm{d}^{\prime}$ intégration sociales. Contrairement aux hypothèses culturalistes, les pratiques et les politiques clientélaires de redistribution doivent être lues dans une optique institutionnelle. Le développement des pratiques clientélaires n'est pas le produit de modèles comportementaux figés inscrits dans une "culture » locale; il est en étroite relation avec la capacité des élus de disposer des ressources publiques et de les distribuer à des segments de populations en quête d'ascension ou de consolidation de leurs positions sociales. Le processus de notabilisation de nombreux élus marseillais est dû à cette capacité du notable de s'ériger en relais essentiel auprès des populations pour que les ressources publiques leur soient destinées dans une logique particulière et personnalisée.

L'analyse de la construction d'une notabilité dans une société urbaine à partir des pratiques clientélaires exige la prise en compte de trois facteurs. Premièrement, la nécessité, consubstantielle au métier d'élu, d'entretenir et d'élargir une clientèle politico-électorale, donc d'alimenter une notabilité à irâvers des pratiques et des attitudes clientélaires. Deuxièmement, l'inscription de l'élu dans un environnement de contraintes territoriales: celles d'une demande sociale incessante d'ordre privé et particulier provenant de son entourage et de la population localisée dans son quartier, son canton, sa circonscription électorale, etc. Troisièmement, la capacité de l'élu de distribuer des biens et des ressources rares aux différents groupes sociaux et aux individus. Nous verrons que les transformations historiques de cette capacité influent lourdement sur le métier d'élu et, par là, sur la construction de la notabilité politique.

6. Je reprends dans cet article des éléments développés dans Mattina (C.), La régulation clientélaire..., op. cit. Les sources utilisées dans ce travail sont essentiellement de deux types : 1) Des dossiers d'archives contemporaines obtenus suite à une dérogation. Ses dossiers contiennent une grande quantité de courriers échangés entre des électeurs, des élus et des techniciens du cabinet du maire de Marseille Ils témoignent clairement des pratiques de recommandations, des services rendus et des logiques de redistribution clientélaires particulièrement dans le domaine des emplois municipaux et des logements sociaux. 2) Une série d'entretiens effectués entre 1999 et 2001 avec des élus de différents niveaux électifs dans le but de comprendre leurs pratiques et leurs représentations face à aux sollicitations de leurs électeurs. 


\section{Métier d'élu et « clientélisation » des électeurs : la notabilisation par les pratiques}

«Quelqu'un qui s'occupe bien des gens et qui en plus réussit un peu ses interventions est bien considéré. Un élu qui s'en fout, et qui tape en touche en disant : "C'est pas à moi", honnêtement il est cuit7. "

En décrivant l'activité du notable français, Pierre Gremion avait déjà remarqué que "l'immense majorité de ses interventions sont soit des interventions portant au niveau des affaires de guichet (les personnes physiques), soit des interventions ostentatoires destinées à affirmer son rôle vis-à-vis de l'électorat ${ }^{8}$ ». Les démarches personnelles, les recommandations, les "pistons", etc., font partie intégrante du métier d'élu. L'étude des courriers adressés par des électeurs et des élus au cabinet du maire de Marseille en vue de l'obtention d'un service ainsi que les entretiens avec des hommes politiques mettent en lumière la dimension du phénomène. Que ce soit dans les années 1970-1980 ou à une époque plus récente, les élus marseillais reçoivent beaucoup de courriers et une grande quantité de personnes les sollicite. A leurs permanences électorales ou dans les différentes institutions dans lesquelles ils ont des mandats électifs, pendant les visites de quartier et dans les différentes manifestations auxquelles ils participent, ils sont constamment sollicités par des personnes venant leur demander une aide, un emploi ou un logement HLM pour eux-mêmes ou pour quelqu'un de leur famille. Même les élus qui, dans leurs discours et dans leurs pratiques, sont le plus opposés aux démarches personnelles savent qu'ils ne peuvent pas ignorer ou "snober " quelqu'un venant leur demander une intervention. Il est très rare qu'ils ne le reçoivent pas ou qu'ils refusent de l'écouter.

Montrer sa disponibilité aux électeurs, les recevoir systématiquement lors des permanences électorales, intervenir en leur faveur en écrivant des lettres de recommandation, etc., tout cela représente pour beaucoup d'élus des activités normales et courantes. La plupart des élus présentent ces activités comme une contrainte dont, même s'ils le voulaient, ils ne pourraient pas se défaire. Certains regrettent ces obligations et n'y voient pas d'alternative ; d'autres les justifient au prisme du rôle social de l'élu. La fonction $d^{\prime}$ « assistante sociale » ou $d^{\prime}$ ' écrivain public », qu'ils mettent souvent en avant pour qualifier certaines de leurs activités, sert à la fois à mettre en évidence cette contrainte qui modifie sensiblement leur rôle et à valoriser leur propre attitude d'écoute et de disponibilité. On retrouve là une véritable

7. Entretien avec un maire de secteur RPR à Marseille.

8. Gremion (P.), Le pouvoir périphérique..., op. cit., p. 236. 
" rhétorique du don de soi " et du sacrifice", qui transparaît clairement dans cet extrait d'entretien avec un conseiller général socialiste de Marseille :

«Les Marseillais, je crois, ils aiment bien le contact avec leur élu, la chaleur, je dirais presque le paternalisme. Ils viennent voir leur élu un peu pour tout et pour rien. Je crois qu'il faut savoir répondre présent, il faut savoir être disponible, il faut savoir sacrifier beaucoup de choses, vie de famille, vie professionnelle, vie associative, pour être à disposition des électeurs et des habitants, je crois que c'est encore plus vrai à Marseille que dans d'autres villes. [...] On est élu aussi pour ça, pour se mettre à l'écoute et à la disposition des gens, c'est un sacrifice. C'est pas toujours facile d'écouter les gens, de 9 heures du matin à 9 heures du soir, mais on est là pour ça. "

Pour répondre de manière systématique aux demandes privées et personnelles, les élus sont obligés d'avoir une organisation performante. Une députée socialiste - vice-présidente de la région Provence-Alpes-Côte$d^{\prime}$ Azur (PACA), conseillère municipale et conseillère $d^{\prime}$ 'arrondissement à Marseille - dispose d'un bureau de permanence personnelle doté de moyens humains importants. Le cumul des mandats locaux et nationaux lui permet d'avoir une équipe élargie de permanents et de bénévoles, qui a la capacité de répondre systématiquement aux demandes des citoyens. Tout courrier est traité, toute personne qui demande un rendez-vous l'obtient assez facilement. Une ligne de téléphone directe avec «votre député » permet un accès simple et rapide à la permanence. Voici comment cette députée nous a détaillé son organisation personnelle :

«Moi, j'ai cinq collaborateurs à plein temps : des collaborateurs qui dépendent de l'Assemblée nationale, sous contrat officiel de l'Assemblée nationale. Puis, j'ai des bénévoles en fonction de leurs compétences. J'ai une bénévole qui vient tenir des permanences une fois par semaine pour les demandes de logement parce qu'elle a des réseaux, etc. J'ai un bénévole sur le social, j'ai un bénévole en matière sportive. Qu'est-ce que j'ai d'autre? J'en ai un ou deux en matière d'éducation et en matière de femmes. Et ils tiennent des permanences. Il y a plusieurs salles, donc, ils reçoivent à un jour fixe, à l'heure fixe, on leur prend des rendez-vous. [...]. Quant aux autres, il y a bien sûr celle qui s'occupe exclusivement de mon planning et des prises de rendez-vous et Dieu sait si c'est un emploi à plein temps. Des filles, il y en a une, (plus une autre à mi-temps) qui tape énormément de courriers, qui assure le suivi, qui fait le double des dossiers, qui archive, etc. Il y a une qui est plus spécialement pointue sur les relations avec l'Assemblée nationale et la culture; et j'ai mon directeur territorial qui s'occupe de lancer le suivi des dossiers. Voilà... »

Les demandes pressantes et continues de la part des électeurs ont amené plusieurs élus à instaurer au sein de leur permanence des structures stables consacrées à la recherche d'emploi. Certains ont créé des associations ou des

9. Lefebvre (R.), « Etre maire à Roubaix. La prise de rôle d'un héritier », Politix, 38, 1997, p. 72. 
cellules spécialisées qui tiennent le rôle de véritables agences d'emploi. Ces structures ont pour charge d'informer les électeurs sur les modalités des épreuves des concours administratifs ainsi que de les mettre en relation avec des entreprises privées susceptibles de les recruter. Un maire de secteur ${ }^{10}$ RPR - qui nous avoue n'avoir jamais pensé, avant d'être élu, devoir s'occuper de questions d'emploi - nous raconte de quelle façon il s'est organisé pour répondre à la demande :

« Moi, je m'occupe beaucoup d'emploi. Donc on a créé deux choses : d'abord, une cellule emploi au niveau de la mairie de secteur qui permet d'informatiser tous les $C V$, de conseiller les gens et de répondre rapidement aux offres qu'on aurait par des entreprises privées ; et nous avons, dans notre secteur municipal [...] créé la première maison de l'emploi. C'est une maison de quartier dans laquelle ne siègent que des associations et des administrations qui touchent à l'emploi. C'est une première à Marseille, et ça a été chez nous. Alors, on a dans ces locaux une ANPE décentralisée, on a des permanences de la Mission locale, des associations [...] qui s'occupent des cadres en difficulté. Tout ça pour montrer, si vous voulez, que même si, dans le cadre administratif pur de la loi PLM, les mairies de secteur n'ont pas de compétences en matière d'emploi, on se donne les moyens de s'en occuper. »

Un autre élu, député-maire de secteur et membre à l'époque de l'entretien de Démocratie libérale, qui a créé une association similaire, nous a décrit en ces termes ses activités :

" J'ai créé une petite association [...] qui gère les demandes d'emploi des gens. On a créé un petit système qui vient en aide aux gens en difficulté. Donc les gens sont reçus et c'est hors de la mairie. D'ailleurs, c'est pas ici, c'est dans mon bureau, dans ma permanence, parce que j'ai une permanence personnelle depuis toujours. Donc là, cette association reçoit les gens, les écoute. On organise par catégories socioprofessionnelles des groupes d'individus et on envoie régulièrement des demandes à des entreprises, en disant: "Voilà, nous disposons de chaudronniers, de..." Voilà. Eux, ils ont $l^{\prime}$ inventaire des demandeurs dans leur spécialité et on a comme ça des résultats qui ne sont pas négligeables. Mais c'est une entreprise bénévole [...]. On fait comme l'ANPE, on envoie les listes de demandeurs d'emploi systématiquement sur les grandes entreprises de la région. Il y en a où je connais ; j'en connais pas beaucoup, eh... Mais, si je sais qu'à tel endroit on fait telle chose, régulièrement, tous les trimestres, on envoie des listes de candidatures. Il s'avère qu'après, finalement, ça fidélise les chefs d'entreprise ou les directeurs des ressources humaines qui, des fois, nous appellent pour dire: "Tiens! Est-ce que vous n'auriez pas un électromécanicien", ou des choses comme ça.»

10. La mairie de secteur regroupe à Marseille deux arrondissements. Il y a à Marseille huit mairies de secteur pour les seize arrondissements de la commune. 
Ces deux exemples illustrent la manière dont l'activité de réponse systématique à la demande privée et personnelle influence fortement les caractéristiques de l'articulation entre le bureau de permanence de l'élu et son mandat institutionnel (local et/ou national). Les demandes d'emploi que l'élu reçoit à sa permanence institutionnelle (à la mairie de secteur dans le premier cas, à la mairie de secteur et au conseil général dans le second) sont transmises aux associations parallèles créées à l'initiative de ces mêmes élus. Ainsi, en ce qui concerne le député-maire de secteur, les demandes d'emploi recueillies dans les institutions sont transmises à une association strictement liée à l'élu, qui n'est rien moins qu'une émanation de son bureau de permanence électorale. Permanents, salariés et bénévoles traitent systématiquement les problèmes et essayent de trouver des emplois pour les demandeurs. Dans le cas du maire de secteur RPR, il n'y a même aucune séparation apparente entre son rôle institutionnel et son activité de réponse à la demande privée et personnelle des électeurs. La création d'une "celluleemploi » auprès de la mairie d'arrondissement répond à l'exigence d'institutionnaliser ce type d'activité et de l'insérer dans un cadre d'action publique en lien avec d'autres institutions s'occupant des mêmes problèmes liés à l'emploi (Mission locale, ANPE). L'institutionnalisation de cette activité d'insertion sert en effet à pallier l'absence de compétence des mairies de secteur en matière d'emploi. Face à la demande pressante de la population, les élus ont jeu facile à justifier la création de structures qui sont de facto illégitimes si l'on s'en thent aux attributions dés minaities. Lciir problème est de réussir à s'imputer le mérite d'une démarche en matière d'emploi. Même la demande d'emploi adressée de manière générique à la mairie doit donc pouvoir être rapportée à une action personnelle du maire en tant que leader politique.

L'attitude de disponibilité et la réponse systématisée aux demandes personnelles et privées s'accordent bien avec une rhétorique, de plus en plus présente dans le discours de beaucoup d'élus, de la présence "sur le terrain " et de la valeur de la "proximité ». Certains parlementaires ne se limitent pas uniquement dans ce domaine à leur propre circonscription; ils répondent aux demandes provenant de tous les arrondissements de Marseille ou même des communes avoisinantes. Le bruit peut se répandre facilement: on sait qu'un député reçoit convenablement, qu'il écoute les gens et qu'il arrive même à obtenir des résultats. Et le public afflue aux permanences. L'action de certains élus ne se limite pas au traitement de la demande reçue de la part de la population; elle peut aller jusqu'à stimuler cette demande et à la rechercher, comme le montre par exemple l'action envers les communautés ethniques et religieuses de Marseille qu'une députée socialiste de la ville nous a décrite en ces termes :

« J'ai des très bons rapports avec les communautés [...] parce que je visite tous les cultes. D'abord, $j^{\prime}$ ai toujours été passionnée par les religions, d'une manière générale $[. .$.$] . En conséquence, moi, le dimanche ou le samedi, je fais$ 
tous les cultes; je fais les mosquées, tous les cultes protestants, je fais toutes les fêtes. Moi, chez eux, je me sens chez moi et ils peuvent se sentir chez eux chez moi. Je trouve ça très intéressant et je pense qu'il est important de prendre du temps à rencontrer les gens dans leur prière et de voir comment ils pratiquent, d'abord... Parce que l'élu a du temps, les gens ont du temps, et puis on se voit avec un regard différent et de temps en temps en dehors de la semaine, c'est plutôt agréable. Et puis, comme les gens sont plutôt repliés sur eux-mêmes, c'est une façon de voir comment ils considèrent la foi, la solidarité, etc. ; et puis, comme ils ne sortent pas, parce que souvent on est enfermé aussi, je vais vers eux. Donc, il est important aussi d'aller vers eux, parce que ces gens-là ils ne viendraient pas aux permanences. Ces gens, vous ne les verrez pas dans la rue forcément [...]. Moi, j'ai trois pagodes bouddhistes, les trois pagodes bouddhistes de Marseille sont chez moi ; ils n'avaient jamais vu un élu de leur vie, eh. Ah, jamais, jamais un élu est allé les rencontrer, je suis la première... »

La recherche et la stimulation de la demande sociale, la réponse systématique à ce type de demande, sont autant d'éléments qui révèlent chez certains élus une attitude de "clientélisation des électeurs». La clientélisation est la mise en œuvre de la part d'un élu d'un ensemble de comportements et de stratégies destinés à afficher une grande disponibilité vis-à-vis des électeurs et à répondre à leurs demandes d'aide concernant des questions privées et personnelles (obtention d'un emploi ou d'un logement social, promotion ou mutation, aide administrative, etc.). L'objectif d'une telle clientélisation est de fidéliser les électeurs : l'élu cherche à créer les conditions pour construire avec chaque électeur un rapport constant et répété dans le temps, fait d'échanges de biens et de services (symboliques et matériels), fondé sur la confiance, l'interconnaissance, parfois sur l'amitié. La clientélisation, la recherche d'un rapport répété dans le temps avec des électeurs à travers des échanges de ressources est un des éléments fondamentaux du processus qui alimente et soutient la notoriété et la notabilité d'un élu.

\section{Les contraintes socio-spatiales de la notabilité :} l'élu face à la demande privée et personnelle

A l'instar de beaucoup d'autres activités de l'élu, celle qui consiste à répondre aux demandes privées et personnelles n'est aucunement séparable de son souci de rélection ${ }^{11}$. Cependant, les intérêts qu'il défend lorsqu'il

11. A propos des maires français, il a été remarqué que « le travail de mobilisation ne s'effectue pas uniquement durant la période électorale précédant les élections mais bien avant. Les élus municipaux et ceux qui aspirent à le redevenir travaillent à se forger sans cesse des réseaux de consolidation et des clientèles durables qui leur permettent plus aisément d'emporter la victoire lors du prochain scrutin et de continuer à jouer " (Le Bohec (J.), "Le travail de mobilisation 
rend un service à l'un de ses proches, à un ami ou à un électeur ne sont pas non plus séparables de ses intérêts affectifs et symboliques à satisfaire les demandes qui lui sont adressées. Si les théories de la professionnalisation politique ${ }^{12}$ et celles qui assimilent les élus à des "entrepreneurs politiques 13 " ont le mérite de saisir des processus de longue durée et de dévoiler les intérêts matériels des élus, elles finissent par négliger d'autres aspects, en particulier l'influence de l'environnement dans lequel ils agissent. L'analyse de la construction d'une clientèle politique demande en effet une analyse localisée du politique ${ }^{14}:$ la prise en compte conjointe de l'action d'un élu et de sa position dans un contexte socio-spatial et historique caractérisé par des contraintes et des opportunités.

\section{Les territoires de la notabilité : la famille Guerini et le quartier du Panier}

Quand on observe de près la scène politique marseillaise, on ne peut qu'être frappé de voir la quantité de familles - essentiellement socialistes - dont les membres font de la politique et occupent, depuis plusieurs générations, des charges électives. Sylvie Andrieux, jeune députée socialiste est la fille d'Antoine Andrieux, ancien adjoint de Gaston Defferre et leader d'envergure de la SFIO puis du PS. Christophe Masse, élu député en 2002 et conseiller général de Marseille, est le quatrième élu consécutif de la famille Miasse après iviarius (éñcôre aujoưrd'hui conseiller général), Jean et un autre Marius, tous deux conseillers généraux avant la seconde guerre mondiale. Dans le seizième canton de Marseille, nous en sommes à la troisième génération de conseillers généraux socialistes appartenant à la famille Weygand (Félix ayant succédé à son père Lucien, lequel avait pris la suite de son père également prénommé Félix). Le poids politique et l'importance historique de ces familles soulèvent des questions quant aux relations entre les parcours notabiliaires, les modalités de construction des clientèles politiques et les milieux partisans (notamment celui du socialisme marseillais) localisées dans des territoires électoraux et sociaux (cantons, secteurs municipaux et quartiers).

L'exemple que nous allons évoquer ici est celui de l'actuel président du Conseil général des Bouches-du-Rhône, Jean-Noël Guerini et de sa famille,

électorale et de dénégation de leur intérêt effectué par les maires ", in Fontaine (J.) Le Bart (C.), dir., Le métier d'élu local, Paris, L'Harmattan, 1994, p. 191).

12. Offerlé (M.), "Professions et profession politique", in Offerlé (M.), dir., La profession politique. XIXe-XXe siècles, Paris, Belin, 1999 ; Gaxie (D.), La démocratie représentative, Paris, Montchrestien, 1993.

13. Garraud (P.), Profession : homme politique, Paris, L'Harmattan, 1989.

14. Briquet (J.-L.), Sawicki (F.), "L'analyse localisée du politique. Lieux de recherche ou recherche de lieux ? ", Politix, 7-8, 1989. 
historiquement implantée dans un territoire situé à proximité du vieux port de Marseille, le quartier du Panier. L'analyse du parcours politique de la famille Guerini, fortement inscrite dans le contexte social et historique de ce quartier, permet de mieux comprendre les processus de sa notabilisation et de la consolidation de « l'éligibilité » de ses membres. Né d'une famille corse du Panier, Jean-Noël Guerini suit les traces de son oncle Jean-François, conseiller général socialiste du troisième canton (qui recoupe essentiellement le Panier) depuis l'après-guerre. Jean-François Guerini a historiquement un rôle important dans le quartier. Commis au bureau d'aide sociale de la municipalité, il avait été à son époque chargé d'enquêter sur les conditions sociales difficiles des immigrants corses et italiens qu'il aidait très souvent à trouver un emploi ou un logement ${ }^{15}$. Dans le sillage familial, Jean-Noël Guerini fait sa carrière politique dans des territoires électoraux dont le Panier est la plaque tournante. En 1982, Jean-Noël succède à son oncle et devient, très jeune, le conseiller général du troisième canton dans lequel, depuis, il a toujours été réélu. A la suite de la mise en place des lois de décentralisation concernant les trois grandes villes françaises (Paris, Lyon et Marseille), il est élu maire d'arrondissement du deuxième secteur ${ }^{16}$ et le reste jusqu'à sa première réélection en 2001 en qualité de président du Conseil général ${ }^{17}$. Le quartier historique du Panier et son canton se trouvent dans le deuxième arrondissement de la ville. Depuis 1977, Jean-Noël Guerini est constamment élu conseiller municipal de Marseille dans ces mêmes arrondissements. A partir du siège de conseiller général du troisième canton, il accède, en 1998, à la présidence du Conseil général, fonction qu'il conserve après les cantonales de 2001 et de 2004. Depuis 1999, il contrôle politiquement la fédération départementale du PS des Bouches-du-Rhône et vise à devenir le candidat de la gauche unie contre le maire actuel de la ville, Jean-Claude Gaudin, aux prochaines municipales.

Posséder des racines locales, hériter du nom d'une famille localement connue, disposer de savoir-faire politiques transmis par le groupe de parenté sont parmi les critères qui, selon Marc Abélès, confèrent une éligibilité territoriale ${ }^{18}$. Mais, pour légitimer et consolider sa position «d'éligible ", l'héritier se doit d'entretenir des réseaux familiaux, amicaux et

15. Attard-Maraninchi (M.-F.), Le Panier, village corse à Marseille, Paris, Editions Autrement, 1997, p. 100.

16. Le deuxième secteur municipal regroupe les deuxième et troisième arrondissements de Marseille.

17. La nouvelle loi sur le cumul des mandats interdit de cumuler le poste de président du Conseil général avec celui de maire d'arrondissement. Jean-Noël Guerini a donc démissionné de son mandat de maire tout en restant conseiller d'arrondissement de ce même secteur, ce qui lui assure une continuité de présence dans les institutions de proximité dans le territoire.

18. Abélès (M.), Jours tranquilles en 89. Ethnologie politique d'un département français, Paris, Odile Jacob, 1989. 
de connaissance, indispensables à la perpétuation de son pouvoir et de celui de sa famille sur un territoire ${ }^{19}$. La perpétuation de l'éligibilité et la conservation du pouvoir de Jean-Noël Guerini se fondent sur trois éléments : l'inscription dans la mémoire historique et politique du quartier ; la continuité historique des pratiques de clientélisation et d'entraide sociale ; la conservation politique et sociale d'un territoire. La continuité de la famille Guerini dans le Panier et dans le troisième canton depuis un demi-siècle se double d'une continuité qui remonte à la fin du XIXe siècle quant à la présence d'élus corses et socialistes dans le même territoire. De double origine, corse et italienne, Henri Tasso est maire de Marseille de 1935 à 1938. Né à Marseille en 1882, après avoir milité dans les Jeunesses socialistes, il adhère à la SFIO en 1905 et devient conseiller général du troisième canton en 1910. En 1924, il devient député de la première circonscription des Bouchesdu-Rhône. Celle-ci regroupe les deux côtés du vieux port et est habitée principalement par des marins, pêcheurs, ouvriers et commerçants d'origine corse et italienne ${ }^{20}$.

Quartier historiquement d'immigration corse et italienne, le Panier est politiquement marqué par des traditions particulières d'exercice de la politique. Marie-Françoise Attard a remarqué, dans ce quartier, la structuration de la politique et des partis autour de l'appartenance au village corse d'origine et du clan entendu comme conglomérat de familles ${ }^{21}$. Plusieurs études sıır la Corse ont montré les relations étroites entre les liens sociaux et familiaux et la formation des groupements politiques 22 . Le pouvoir politique des familles corses sur le quartier, la transmission par voie d'héritage des mandats politiques sont autant d'éléments qui s'inscrivent dans une longue durée et s'entrecroisent constamment avec la mémoire et la pratique des services rendus entre la population et la famille politique dominante. Jean-Noël Guerini ne fait que renouer constamment avec la mémoire et la tradition de sa famille et des Corses du quartier lorsqu'il reçoit, dans sa permanence de la place Francis Chirat, des dizaines et dizaines de personnes, à l'aide de ses assistants et collaborateurs, afin de recueillir et de traiter leurs demandes. Il s'inscrit ainsi dans une attitude de disponibilité et de clientélisation des électeurs. Les échanges de courriers conservés dans les archives municipales témoignent d'ailleurs de son activité constante, depuis les années 1980, d'intervention et de

19. Selon J.-L. Briquet, le notable se doit d'attester de la présomption de notabilité qui est lui est conférée par sa position sociale et familiale au travers de conduites particulières (échanges clientélaires, ethos de la proximité, de l'amitié, etc.) (Briquet (J.-L.), La tradition en mouvement. Clientélisme et politique en Corse, Paris, Belin, 1997).

20. Sur la vie d'Henri Tasso, cf. Olivesi (A.), « Henri Tasso, 1880-1944 », Marseille, 100, 1975.

21. Attard-Maraninchi (M.-F.), Le Panier..., op. cit.

22. Cf., entre autres, Lenclud (G.), «Des idées et des hommes. Patronage électoral et culture politique en Corse ", Revue française de science politique, 38 (5), 1988. 
recommandation auprès du cabinet du maire en vue d'intervenir en faveur de ses électeurs. Au début des années 1980, Jean-Noël Guerini, jeune conseiller municipal et général, intervient habituellement auprès du cabinet du maire et de l'OPAC Sud (organisme HLM lié au Conseil général des Bouches-du-Rhône) pour signaler des personnes lorsqu'il y a des vacances d'appartements dans les immeubles du Panier. Il lui arrive souvent d'obtenir satisfaction en faisant attribuer des logements à ses protégés. Une correspondance, datée de 1981, entre Jean-Noël Guerini, le cabinet du maire et le président de l'OPAC fait apparaître les termes de l'échange entre la famille Guerini et l'entourage du maire. Le secrétaire particulier du maire, V. M. écrit ainsi à S. O., directrice du cabinet :

«Monsieur D. résilie le bail de l'appartement qu'il occupe au 36, esplanade de la Tourette (2e). Jean-Noël Guerini souhaiterait que ce logement soit affecté à Madame S., domiciliée [...] [dans le même secteur]. Je vous précise que j'interviens de mon côté auprès de $M$. Masse et $M$. Weygand ; je vous serais donc reconnaissant de bien vouloir faire le nécessaire auprès de $M$. Faure ${ }^{23}$ [président de l'OPAC]. »

Suite à cette lettre, Mme S. O. intervient auprès du président de l'OPAC par une lettre « très signalée » pour que satisfaction soit donnée à la personne en question, en ajoutant dans la lettre la mention: «Le Maire y tient beaucoup. » L'intervention produit ses effets. Il ne passe pas plus d'un mois et le logement est attribué par le président de l'OPAC:

«J'ai le plaisir de vous informer que, suite à mon intervention, un appartement a pu être attribué à l’Esplanade de la Tourette à Mme S. J'espère avoir apporté toute satisfaction 24 [...]. »

Recevoir longtemps les électeurs dans le quartier, être présent aux fêtes du troisième âge organisées par une association de solidarité intergénérationnelle dans les mêmes locaux que sa permanence électorale, multiplier les visites de quartier, etc., veut dire pour Jean-Noël Guerini marquer matériellement et symboliquement une présence et une action liées à l'entraide, à l'assistance sociale, à la solidarité, etc., qui renouent avec les préoccupations politiques de son oncle, ancien conseiller général du même canton. Cela prolonge et confirme son enracinement dans la société de son quartier de naissance et d'appartenance.

Le maintien de l'éligibilité et la continuité de l'exercice du pouvoir dans un quartier de tradition populaire corse et italienne, passent aussi par la conservation de l'identité et de l'histoire de ce territoire. La recherche d'une continuité politique de Guerini au Panier est allée de pair avec une tentative

23. Archives municipales de Marseille, série 599W9, demandes HLM, 1980-1982.

24. Archives municipales de Marseille, série 599W9, demandes HLM, 1980-1982. 
de conservation de l'identité du quartier alors, qu'en même temps, la composition de sa population changeait radicalement. Depuis les années 1970, le dépeuplement du Panier est dû principalement au départ progressif $d^{\prime}$ une bonne partie de l'ancienne population d'origine européenne (corse, italienne, arménienne) qui accomplit son parcours d'ascension sociale en se déplaçant vers les appartements plus spacieux et confortables des quartiers plus aisés ${ }^{25}$. Elle est remplacée par des populations d'origine maghrébine, puis comorienne, attirées par les prix relativement bas des appartements. Toutefois, les politiques publiques de réhabilitation du quartier $s^{\prime}$ inscrivent dans une logique de sauvegarde et de conservation. La politique de rénovation immobilière du Panier a débuté dans les années 1970, à partir de l'idée d'un groupe d'artistes et de promoteurs immobiliers qui souhaitaient réhabiliter l'habitat et faire de ce quartier un petit "Montmartre marseillais ", avec la volonté affichée de favoriser le maintien sur place des anciennes familles de pêcheurs italo-corses ${ }^{26}$.

L'enracinement social et politique de Jean-Noël Guerini et de sa famille dans ces quartiers, son statut d'éligible et son parcours de notabilisation nous permettent ainsi d'observer ses pratiques clientélaires sous un autre jour. L'attitude d'un élu face à la demande personnelle et privée des électeurs, sa propension plus ou moins grande à répondre, à faire systématiquement des interventions, ne dépend pas uniquement ni principalement d'une stratégie itilitariste visant à obtenir pluıs de voix sur un marché électoral. La propension à la proximité avec les électeurs doit être considérée en lien étroit avec des processus d'apprentissage des savoir-faire politiques inscrits dans des contextes familiaux et dans des territoires historiquement enclins à la culture de l'entraide. Dans des villes populaires comme Marseille, caractérisées par une pauvreté relative, par un chômage élevé depuis les années 1980, par les aléas et l'insécurité du travail portuaire et dans l'industrie locale, la recherche d'un emploi public et d'un logement social a représenté dans les années de l'après-guerre une grande opportunité $d^{\prime}$ 'intégration et $d^{\prime}$ ascension sociale pour une partie du prolétariat et la petite bourgeoisie locale. La pression de la population vers les élus et notables marseillais a donc toujours été très soutenue dans ce domaine. Elle s'est exprimée par les différents registres de la revendication, de l'appartenance et de la citoyenneté, témoignant de la construction populaire d'une politique

25. Mazzella (S.), Zalio (P.-P.), Cinquante ans de recherche urbaine à Marseille. Bilan bibliographique (1954-1995), Etude pour le Plan urbain, 1996.

26. Picon (B.), " "Protection" et "réhabilitation". Crises de croissance (le parc naturel régional de Camargue, le quartier du Panier à Marseille) ", Cahiers de l'Observatoire du changement social, IX, 1982; Mattina (C.), "L'intermédiation politique des présidents de Comités d'intérêt de quartier : le territoire de la notabilité ", in Fournier (P.), Mazzella (S.), dir., Marseille entre ville et ports. Les destins de la rue de la République, Paris, La Découverte, 2004. 
par le bas ${ }^{27}$ qui est aussi une manière de contraindre les élus à répondre aux attentes de leurs électeurs.

\section{La demande privée et personnelle : les registres clientélaires de l'appartenance}

"J'ose penser que s'il est normal que tout militant devait soutenir le parti dans l'adversité, c'est aussi normal que quand celui-ci est victorieux, il pense à accorder une faveur à ceux qui lui sont fidèles 28 . »

Face à un système de fonctionnement de l'administration marseillaise perçu comme fortement injuste à l'avantage de personnes recommandées, la recherche d'un " piston " est souvent considérée par les électeurs comme le seul vrai moyen d'obtenir satisfaction. L'idée qu'il existe un favoritisme systématique dans le domaine du recrutement à la mairie et dans les autres collectivités locales ou dans l'attribution des logements HLM est fortement présente dans la correspondance que les Marseillais adressent au cabinet du maire dans les années 1970-1980. Une employée de l'hôpital de Créteil, qui a demandé une mutation à l'Assistance publique de Marseille et dont la candidature a été rejetée, écrit au maire de Marseille, par ailleurs ministre de l'Intérieur à l'époque, en ces termes :

"M. le Ministre, permettez-moi de vous traduire ma profonde tristesse morale et mon étonnement à constater que l'on puisse, avec une certaine désinvolture, rejeter ma requête. Car, je sais, que les hôpitaux sans recruter de façon outrancière ont au compte goutte parfois, une ou deux possibilités de recrutement toujours réservées aux personnes les mieux recommandées [...]. Je me vois contrainte de vous dénoncer personnellement ce que je considère être une injustice sociale. Je n'ai pas la chance d'être l'une des vos parentes ni de vos amies, mais je vous supplie d'agir comme si cela était. Vous seul, par une intervention directe, pouvez apporter une solution à mon problème ${ }^{29}$. "

Dans les archives, on retrouve un grand nombre de lettres adressées au cabinet du maire par des personnes demandant un service ou revendiquant le rétablissement de leurs droits au prisme de leur appartenance politique, familiale ou ethnique. Plusieurs courriers de personnes se déclarant militants socialistes témoignent de la protestation contre le favoritisme et l'injustice du système; mais ils contiennent en même temps des demandes de services impliquant un "coup de pouce » sur un dossier au regard de

27. Bayart (J.-F.), Mbembe (A.), Toulabor (C.), La politique par le bas en Afrique noire. Contribution à une problématique de la démocratie, Paris, Karthala, 1992.

28. Il s'agit des phrases extraites d'une lettre d'un militant socialiste et adjoint des cadres hospitaliers qui demande au maire un appui pour une promotion dans les services de l'hôpital (Archives municipales de Marseille, série 802W34, dossiers particuliers, 1980-1983).

29. Archives municipales de Marseille, série 802W34, dossiers particuliers - cabinet du maire, 1980-1983. 
leur loyauté et de leur fidélité au parti socialiste. Voici par exemple la lettre de quelqu'un se revendiquant militant socialiste et qui demande au maire d'embaucher son neveu au service d'assainissement de la ville de Marseille :

"Je sais bien qu'il n'y a pas d'embauche, mais je pensais que vous m'auriez donné un espoir, compte tenu que prochainement le recrutement reprendra à la mairie et vous le savez mieux que personne, car, que je sache vous êtes le "patron". Vous devez me rendre justice, dans 33 ans de parti je n'ai jamais rien demandé pour les miens, aussi je croyais que vous auriez un peu d'amitié pour moi qui me suis si souvent battu à vos côtés. Je sais et je crois que vous n'êtes pas au courant que pour d'autres les choses sont possibles. Je ne pense pas que vous ayez un reproche à me faire car j'ai toujours été loyal. [...] Je dois dire que malgré une réponse négative de votre part, je resterai le socialiste que je suis depuis 33 ans "bête et discipliné", mais malgré tout amer de voir qu'il y a plusieurs poids et mesures ${ }^{30}$."

Les revendications sont étonnantes par leur complexité, parfois par leurs contradictions, et par le mélange de différents registres de représentation de l'autorité, de protestation, de justification et d'appartenance. En même temps qu'on critique un fonctionnement comme injuste, on réclame la réparation de l'injustice par l'emploi des mêmes pratiques de favoritisme que l'on dénonce et que l'on condamne en principe. L'injustice est vue au prisme d'une situation personnelle et subjective dans laquelle l'individu a la sensation de subir un tort et de ne pas être pris en considération par des autorités politiques qui, pourtant, devraient lui être reconnaissantes. Dans ie courrier qui vient d'être cité, comme dans beaucoup d'autres du même type, la demande de faveur personnelle montre que l'on ne peut que très difficilement départager les deux facettes de la même médaille : la recherche de la satisfaction d'un intérêt matériel et la reconnaissance affective et symbolique $^{31}$. Obtenir satisfaction à une demande de faveur est aussi une reconnaissance pour le travail de militant et pour la fidélité envers le parti.

Dans plusieurs courriers d'électeurs et de militants, on retrouve différentes formules de présentation de soi qui servent à appuyer les demandes auprès des autorités publiques. Les demandeurs cherchent en effet à faire jouer auprès de l'homme politique les atouts personnels ou familiaux qu'ils pensent pouvoir utiliser pour toucher davantage sa sensibilité ou susciter son intérêt. Plusieurs registres d'appartenance s'entrecroisent constamment :

30. Archives municipales de Marseille, série 802W38, dossiers particuliers - cabinet du maire, 1980-1983.

31. Dans sa recherche sur le clientélisme politique en Corse J.-L. Briquet était arrivé à la même conclusion : "La dimension matérielle de l'échange clientélaire n'est pas occultée, mais elle ne peut transparaître légitimement que comme la conséquence de sentiments qui la transcendent (la "fidélité" et la reconnaissance) tout en la rendant possible " (Briquet (J.-L.), La tradition en mouvement..., op. cit., p. 24). 
les électeurs peuvent valoriser leurs liens familiaux ou amicaux avec l'élu ou quelqu'un de son entourage ; ils peuvent revendiquer leur appartenance au parti, à la mairie ou à un corps de l'administration de l'Etat pour lequel ils supposent que l'élu se montrera plus attentif; ils peuvent également rappeler à l'élu leur appartenance à une communauté ethnique composant la ville, comme celle des Corses ou des Arméniens. Un courrier d'une militante socialiste et attachée communale contractuelle, écrivant au maire pour solliciter sa titularisation, témoigne des registres d'appartenance à la fois partisane (au PS), institutionnelle (à la mairie) et ethnique (à la " communauté » arménienne) :

"C'est avec une grande joie que ma famille et moi-même avons accueilli, dimanche 13 mars [1983] la nouvelle de votre réélection à la mairie de Marseille et vous adressons à cette occasion nos plus chaleureuses félicitations et nos vœux de réussite [...]. Contribuant pour ma part, de toutes mes forces, à cette entreprise, j'ai notamment préparé, en tant que militante du PS (16e section), les visites d'immeubles effectuées par Mme Charles-Roux Defferre dans le septième arrondissement. Je me permets aujourd'hui d'attirer votre attention sur ma situation personnelle. Depuis 1981 je suis attachée communale contractuelle à la mairie de Marseille. Tout en travaillant et malgré le peu de temps libre consacré aux révisions [...], je me présente pour la première fois au concours d'attaché communal. [...] Aux épreuves orales, trois points me feront malheureusement défaut pour pouvoir être inscrite sur la liste d'aptitude aux fonctions d'attaché communal. Remise de ma tristesse et de ma profonde déception et connaissant vos grandes qualités de cœur et votre bonté, je prends aujourd'hui la liberté de solliciter de votre haute bienveillance la titularisation que j'aurais dû obtenir par la voie de concours. Je ne sais si ma requête recevra un accueil favorable et si l'exposé de mon cas personnel a pu vous émouvoir. Néanmoins, en tant que membre d'une nombreuse communauté arménienne de Marseille, descendant d'un peuple poursuivi par les guerres et victime des massacres, je suis persuadée que vous êtes, Monsieur le Maire et Monsieur le Ministre de l'Intérieur, le plus ardent défenseur de notre intégration dans la sauvegarde de notre identité culturelle 32 [...]. »

Ces courriers montrent que la demande d'un service ou d'une faveur n'implique pas uniquement chez ses électeurs des considérations utilitaristes, dépolitisées et dénuées de tout sens civique. Il dévoile aussi des processus d'identification collective $\mathbf{e}^{33}$ : le sens d'appartenance à un parti, à une famille, à une communauté ethnique, à un groupe syndical, à une corporation, à une administration. Si l'on dépasse le regard moralisateur sur

32. Archives municipales de Marseille, série 802W32, dossiers particuliers - cabinet du maire, 1980-1983.

33. Pizzorno (A.), «Sur la rationalité du choix démocratique », in Birnbaum (P.), Leca (J.), dir., Sur l'individualisme, Paris, Presses de la FNSP, 1991. 
une attitude utilitariste et cynique d'électeurs proposant un marchandage autour du vote ou du soutien politique aux élus, on peut considérer ces démarches comme autant de modalités possibles d'appropriation sociale et individuelle de l'expression du vote et de l'engagement partisan. Le fait de solliciter un élu pour une faveur personnelle ou pour un passe-droit n'est pas forcément ressenti par les électeurs eux-mêmes comme une action illégitime. Il peut être présenté et conçu comme la revendication tout à fait légitime d'un droit ou comme la réparation d'un tort ou d'une injustice. Des citoyens qui ne sont pas forcément très politisés participent à la politique et aux processus démocratiques avec les codes, les langages et les méthodes qu'ils sont capables d'exprimer par rapport à la perception qui est la leur du métier d'élu, de l'autorité publique et des institutions auxquelles ils s'adressent.

En même temps qu'ils expriment une revendication ou qu'ils réclament la réparation d'une injustice, ces électeurs peuvent utiliser le vote comme une promesse ou comme une menace de sanction pour l'élu. L'électeur peut écrire à un élu ou aller le voir en lui promettant qu'il votera (avec toute sa famille) pour lui. Toutefois, d'une part il pourra aller voir plusieurs élus et d'autre part il restera, dans la plupart des cas, libre de son choix lorsqu'il sera dans l'isoloir. L'élu, pris dans ce qu'il perçoit comme une obligation de répondre aux demandes personnelles des électeurs mais en même temps jamais véritablement assuré que ce travail de terrain lui rapportera des fruits à la sortie des urnes, apparâit ainsi dans une situation fragile. Comme l'a montré Alain Garrigou, l'élargissement du suffrage a introduit un élément $d$ 'incertitude chez les notables, lesquels ont été de plus en plus contraints de solliciter le vote des électeurs et d'accomplir un travail spécifique pour remporter des élections : « Le client, désormais fort de son bulletin de vote, peut éventuellement marchander son soutien au patron ${ }^{34}$. " Il s'en est suivi une relation plus égalitaire entre élus et électeurs, en tout cas une relation très éloignée du rapport de dépendance économique et sociale tel qu'il a été décrit à propos du clientélisme politique dans les sociétés rurales, par exemple les communautés paysannes du monde méditerranéen ${ }^{35}$. Dans les milieux urbains, ainsi que le montre le cas de Marseille, l'échange clientélaire a été pour certains électeurs un moyen d'exercer une contrainte

34. Papadopoulos (Y.), Vaner (S.), « Le clientélisme de parti », Cahier du CEMOTI, 7, 1989, p. 8. 35. Selon un accord qui s'est produit dans les différentes disciplines des sciences sociales entre les années 1960 et 1970 , l'inégalité entre le patron et le client dériverait du contrôle largement déséquilibré des ressources sociales (de statut et d'influence), politiques (de pouvoir) et économiques (de richesse). Cf. Briquet (J.-L.), "La politique clientélaire. Clientélisme et processus politiques », in Briquet (J.-L.), Sawicki (F.), dir., Le clientélisme politique dans les sociétés contemporaines, Paris, PUF, 1998. Pour une définition classique du clientélisme politique, cf. Médard (J.-F.), «Le rapport de clientèle. Du phénomène social à l'analyse politique ", Revue française de science politique, 26 (1), 1976. 
sur les élus, à travers les demandes sociales incessantes qu'ils leur adressaient et auxquelles ces élus étaient en quelque sorte sommés de répondre. La construction et l'entretien de la notabilité répondent ainsi à des contraintes fortes, en particulier la possibilité pour les élus de satisfaire les exigences de leurs électeurs. S'il est vrai que l'élu qui réussit à fidéliser un électorat sur une base clientélaire a des réelles opportunités d'enracinement local et de carrière, il reste que cet élu est l'objet d'une demande sociale constante qu'il ne peut toujours satisfaire. De la tension entre ces deux facteurs dépendent les modalités de construction et de maintien de la notabilité politique.

\section{La transformation des ressources clientélaires :}

\section{de l'évergétisme notabiliaire à la médiation symbolique}

A travers la notion d' "évergétisme" Paul Veyne a repéré, dans la propension des hommes incarnant l'autorité à faire état de leur générosité et à faire des dons, l'un des fondements essentiels de leur pouvoir au sein de la société romaine ancienne ${ }^{36}$. Tantôt les évergésies étaient offertes par les notables en dehors de toute obligation définie (l'évergétisme libre), tantôt elles étaient offertes à l'occasion de leur élection à un " honneur " public, à une magistrature ou fonction municipale (l'évergétisme ab honorem). Tout en gardant à l'esprit la distance qui sépare des époques et des contextes sociaux très éloignés, on ne peut éviter de remarquer que de telles pratiques évergétiques demeurent des éléments fondamentaux de la construction du leadership politique et de la légitimation de la notabilité dans les sociétés modernes. Les pratiques de clientélisation des électeurs, la construction des notabilités, sont liées à la possibilité que les élus possèdent d'influencer des décisions, de gérer de l'argent public, d'allouer des fonds à certaines catégories et groupes sociaux, de faire attribuer des emplois, des logements, des subventions à tels ou tels individus ou familles. Mais qu'en est-il de ces élus et de ces notables lorsque leur efficacité à satisfaire la demande sociale $s^{\prime}$ affaiblit ? Surtout, comment se modifient leur rôle et leur fonction lorsque les ressources traditionnelles qu'ils pouvaient distribuer de façon discrétionnaire se rétrécissent considérablement? Ces questionnements se posent obligatoirement à partir du moment où, dans les années 1980, à Marseille (comme d'ailleurs dans d'autres grandes villes européennes), l'intégration et l'ascension sociale par l'accès aux emplois publics et au logement social perdent de leur importance et de leur efficacité.

36. Veyne (P.), Le pain et le cirque. Sociologie historique d'un pluralisme politique, Paris, Le Seuil, 1976. 


\section{Emplois publics et logements sociaux : les capacités de redistribution comme ressources notabiliaires}

Dans la période de grande expansion démographique de Marseille et d'arrivée de nombreuses populations immigrées dans la ville (les années 19551975), les politiques de redistribution des ressources comme l'emploi, le logement social et les aides sociales personnalisées ont été au centre de l'action municipale et étatique. Dans cette phase, deux domaines en particulier, ceux de l'emploi et du logement, ont représenté autant de facteurs fondamentaux dans l'amélioration de la vie des populations et dans les parcours de mobilité sociale individuelle et collective. A Marseille, les pouvoirs publics - en particulier la municipalité - ont pu disposer de grandes facilités dans l'attribution de ces ressources. Au milieu des années 1960, la mairie disposait directement et indirectement d'environ 50000 emplois: les emplois municipaux, de la Régie des transports marseillais (RTM), de certaines entreprises du port, de sociétés de taxis, de sociétés d'économie mixte, les emplois publics et parapublics d'organismes dont elle contrôlait les conseils d'administration (par exemple les hôpitaux de l'Assistance publique, dont la présidence est assurée par le maire), ceux d'organismes sociaux proches du syndicat Force ouvrière, des offices $\mathrm{HLM}^{37}$. A l'instar d'autres villes, le cabinet du maire de Marseille a toujours eu une grande latitude en matière de distribution des emplois publics municipaux C'est par le cabinet du maire que passaient officiellement les recrutements airects - ceux quii ne faisaicnt pas l'objet d'un concours - à la mairie et dans tous ses services rattachés. Son pouvoir était d'autant plus important qu'une forte pression sociale était exercée par la population sur des emplois à basse ou à très basse qualification, comme ceux d'éboueur, de cantonnier, de brancardier, de fossoyeur, de cantinière, etc. Le fait qu'il y ait eu une grande quantité de personnes aspirant à ces emplois et se présentant à des concours de recrutement contribuait en effet à renforcer le caractère arbitraire des choix et à favoriser les embauches de type clientélaire. Le maire et son cabinet exerçaient en outre un pouvoir fondamental dans le domaine des conditions de travail des employés communaux et paracommunaux : affectations, déplacements, promotions, attributions de titres honorifiques, etc., étaient autant d'occasions de satisfaire les requêtes et les exigences de ce large public municipal. Ces attributions donnaient ainsi au maire et à son cabinet un vrai pouvoir sur les parcours d'ascension sociale des individus et des familles ${ }^{38}$.

37. Sanmarco (P.), Morel (B.), Marseille. L'endroit du décor, Aix-en-Provence, Edisud, 1985, p. 106-107.

38. Les dossiers d'archives sur lesquels nous avons travaillé laissent apparaître une grande quantité de lettres de recommandation qui concernent non seulement le domaine de l'embauche mais aussi les modalités de mobilité (promotions, nouvelles affectations de poste, changement de services, etc.) à l'intérieur de la mairie. 
Dans le domaine de l'attribution des logements sociaux, les possibilités discrétionnaires de redistribution des ressources étaient tout aussi importantes. Les échanges de courriers entre le cabinet du maire et le service logement de la mairie pour la période 1970-1986, les listes d'attribution des logements sociaux, les notes manuscrites des élus et des techniciens, nous permettent de reconstruire les pratiques et les arrangements en matière d'attribution de ce type de logements. $\mathrm{Si}$, formellement, ce sont les commissions d'attribution des sociétés HLM qui détiennent le véritable pouvoir de décision, les pratiques repérées dans les sources d'archives et les témoignages d'acteurs montrent autre chose : les commissions d'attribution étaient à cette époque des chambres de pure et simple ratification de choix décidés en amont par la négociation et la médiation informelle entre les différents acteurs du logement social (élus, techniciens de la mairie et des organismes HLM, représentants des associations de locataires). Toutefois l'ample marge de manœuvre dont les élus disposaient nécessitait aussi des règles informelles de partage des ressources afin de limiter les possibles conflits. C'est ainsi dans le registre de l'informel que se sont créés des critères de partage des logements à attribuer entre le cabinet, les groupes politiques et les conseillers municipaux. Les courriers au sujet de la mise en location d'appartements dans le groupe HLM «Saint Barthélemy » nouvellement implanté dans le quatorzième arrondissement et les listes d'attribution pour la période comprise entre avril 1969 et juin 1970 classées, dans des chemises bien séparées, par groupement politique, montrent la formalisation de ce partage par des quotas préétablis de logements ${ }^{39}$. Les 717 logements sociaux attribués par la municipalité dans la cité Saint Barthélemy pendant ces quatorze mois, ont été partagés ainsi entre les différents groupes politiques : 187 logements pour le maire de Marseille (et son cabinet); 46 pour les fonctionnaires municipaux ; 71 pour le service de logement de la mairie (et donc contrôlés par l'adjoint au logement); 313 pour les groupes politiques soutenant la majorité municipale (répartis en 182 pour la SFIO, 53 pour le groupe "Socialisme et démocratie", 111 pour le groupe "Républicain d'union et d'action communale » et 67 pour le groupe «Centre démocrate »40.

L'attribution des logements sociaux se fait donc par un accord préalable entre le cabinet du maire, l'adjoint au logement et les quatre groupes de la majorité defferrienne, résultat de l'alliance entre les socialistes et les partis centristes. Chacun de ces acteurs possède la faculté d'attribuer des logements aux personnes ou aux familles de son choix. En outre, dans l'ensemble des logements dont chaque groupe politique dispose, chaque conseiller municipal possède un quota personnel de logements à attribuer.

39. Archives municipales de Marseille, série 540W24-26, listes d'attribution des logements sociaux - office HLM, archives Rastoin, 1966-1970.

40. Ibid. 
Nous avons pu trouver les listes des contingents pour chaque conseiller municipal du groupe SFIO avec un nombre de logements qui varie selon les cas de deux à cinq. Un logement bien précis (par exemple, tel appartement situé dans telle tour de telle cité HLM) est mis à disposition d'un élu qui en désigne le bénéficiaire en cas de vacance (départ du locataire précédent, rejet $d^{\prime}$ une candidature). De manière très routinière, l'élu désigne son propre candidat à un appartement en adressant sa candidature au cabinet du maire. On peut citer à ce sujet deux exemples typiques et très récurrents dans nos dossiers. Il s'agit de deux lettres adressées par des élus du groupe «Socialisme et démocratie » aux fonctionnaires du cabinet :

« Je t'adresse le dossier de Mme $X$, mère de famille qui a été expulsée pour la fin du mois. Je lui attribue un type 3 [appartement de trois pièces] à St. Barthélemy qui a été mis à ma disposition41. "

« Je t'adresse sous ce pli le nom de la personne que je désigne pour le type 5 de St. Barthélemy en remplacement du nommé $X$ qui a été rejeté42. "

Le langage de ces courriers, ainsi que le système de partage par groupe et par conseiller municipal, montrent que ces élus se comportent comme si les logements rentrant dans leurs quotas leur appartenaient personnellement. Il y a là comme une véritable appropriation d'un bien public à travers une interprétation extensive des pouvoirs des élus, des compétences et attributions institutionnelles dérivant de ces pouvoirs. L'existence de ce système de quotas par groupe et par élu est confirmée par des entretiens avec certains memures de la majorité socialiste et communiste entre les années 1983 et 1989, qui dessinent un système $d^{\prime}$ arrangement finalement très consensuel :

"Voilà : il y avait des contingents par groupe politique et y compris à l'intérieur des groupes politiques... Voilà moi, $j$ 'ai trois candidats, vous en avez cinq... En fonction des promesses que nous avions faites et en se disant : si on rend service à cette famille, là on prend vingt voix $d^{\prime}$ un coup ${ }^{43}$. "

"On dit, voilà, dans tel groupe il y a trente logements. Sur ces trente logements vous en avez cinq pour le PC, donc on envoie les clients pour chacun des logements. Une fois qu'on arrive dans la commission [d'attribution des logements sociaux], si les services ont respecté les instructions, la question est terminée, c'est attribué. De temps en temps, vous allez avoir un représentant des locataires qui va crier, à ce moment-là on lui donne un logement, pour la prochaine fois, voilà. Ça se passe de manière très consensuelle... Puisque la mécanique c'est d'être redevable et de protéger son client, on ne va pas faire des affrontements politiques ${ }^{44}$. "

\section{Ibid.}

42. Ibid.

43. Entretien avec un ancien adjoint PS de Defferre à la mairie de Marseille.

44. Entretien avec un conseiller général PC de Marseille. 
Le pouvoir du maire dans la redistribution des ressources clientélaires ne réside pas uniquement dans la satisfaction des demandes de ses clientèles personnelles et de son entourage. Ce pouvoir est d'autant plus grand que le maire dispose de ressources publiques et qu'il a la possibilité de les redistribuer à travers les différents leaders et conseillers municipaux appartenant à son équipe. La distribution des ressources est l'une des caractéristiques principales de l'emprise qu'un leader a sur son entourage. A la suite de Frederick Bailey, qui a observé la formation du leadership dans la société indienne, on peut donc dire que le leader est celui qui gère et distribue des ressources dans une certaine situation politique ${ }^{45}$. Le pouvoir notabiliaire et sa reproduction sont donc étroitement liés à la capacité du notable de participer à un système d'échanges avec les électeurs et les groupes qui le soutiennent ${ }^{46}$. La transformation et, en l'occurrence à Marseille, le rétrécissement des possibilités de redistribution des ressources clientélaires classiques comme les emplois et les logements sociaux finiront, de manière inévitable, par transformer le leadership notabiliaire, le prestige dont il était investi et les pratiques sur lesquelles il se fondait.

La notabilité face au rétrécissement des ressources clientélaires : la réponse symbolique des élus

Dans des périodes d'expansion économique et démographique, de plein emploi, d'accession au travail et au logement d'un grand nombre de familles et d'individus, les canaux clientélaires ont souvent représenté un moyen de socialisation des individus à la politique et aux milieux partisans locaux, $d^{\prime}$ intégration et d'ascension sociale de couches populaires ainsi qu'un moyen d'amélioration de leurs conditions d'existence. Toutefois, à partir de la deuxième moitié des années 1970, on assiste graduellement à une modification de cette situation. Au début des années 1980, sur le fond d'un mouvement de dépeuplement de Marseille (qui passera de 920000 habitants au milieu des années 1970 à 800000 environ en 1990), on assiste à une croissance du chômage, au départ hors des logements sociaux des populations issues de la petite et de la moyenne bourgeoisies et à la dégradation progressive des cités. Les constructions d'immeubles HLM reculent fortement à partir de $1975^{47}$; la mairie diminue sensiblement les

45. Bailey (F. G.), Les règles du jeu politique, Paris, PUF, 1971.

46. Briquet (J.-L.), La tradition en mouvement..., op. cit., p. 195.

47. Si l'on suit le rythme des mises en location d'appartements HLM, on voit clairement chuter le nombre d'appartements entre les années 1960 et les années 1980 . Le pourcentage de mise en location d'appartements HLM sur le total des mises en location dans la commune de Marseille passe de $32,3 \%$ dans la période $1968-1974$ à 5,1 \% entre 1981 et 1986. (Direction départementale 
embauches, une volonté politique et technocratique se met en place pour assainir les budgets des institutions publiques et pour modifier des comportements anciens ${ }^{48}$.

A Marseille, les possibilités de satisfaire concrètement les demandes privées et personnelles des citoyens se font de plus en plus rares. Cela ne veut pas pour autant dire que les pratiques et les échanges clientélaires disparaissent, mais il se crée, à cette époque, une situation de tension contradictoire. Les élus ne disposent plus comme auparavant d'une grande quantité de biens à distribuer de façon discrétionnaire à la population; mais les habitudes et les pratiques d'échange clientélaire se sont, tout le long des années 1960 et 1970, fortement ancrées dans la société urbaine marseillaise. Ainsi, les élus continuent à recevoir les habitants et à leur faire des recommandations et des lettres de soutien. Toutefois l'efficacité réelle de ces démarches est de moins en moins assurée. Les entretiens avec les élus marseillais les plus âgés mettent en relief comment ils ont perçu un tel changement :

« Avant chaque élu avait la capacité de donner. [...] Un électeur venait et disait à l'élu : "Vous savez, moi, je suis d'une grande famille, si vous réglez le problème de mon fils, on ne sera pas ingrats, etc." C'est comme ça que ça se passait. Et puis l'élu décrochait le téléphone, il appelait la société de construction Untel et il disait: "Vous savez, je vous ai donné quand même trois marchés, vous m'embauchez un tel, un tel et un tel." Tout ça fonctionnait Bien ou mal, tout ça fonctionnait. [...] Les gens étaient omnipotents. C'est fini aujourd'hui [...] Aujourd'hui, il y a crise de l'emploi. L'élu qui prétendrait avoir une solution individuelle au problème de l'emploi est obligé de se faire carboniser! Parce que si même il a une réponse pour un individu, il va dire non à tous les autres et il va se carboniser ! Le logement à Marseille c'est fini, il y a un parc immobilier dont un cinquième de logements sont vides. Personne n'en veut ${ }^{49}$. "

Questionnés sur leur attitude face aux demandes personnelles des électeurs, certains élus marseillais se montrent prudents : si, d'un côté, ils affichent une grande disponibilité à écouter ces demandes, ils tiennent d'autre part à préciser aux électeurs qu'ils ne peuvent pas faire de promesses et qu'ils ne sont pas tenus par une obligation de résultat. Donc, lorsqu'un élu est sollicité par un électeur pour obtenir un emploi, il entame dans la plupart des cas des démarches : il écrit, selon les différents cas, aux présidents des collectivités territoriales ou aux PDG de certaines entreprises privées pour recommander la candidature de la personne intéressée. Toutefois, il est conscient de la pression existante dans le domaine de l'emploi. Il sait également que tous ses

de l'équipement des Bouches-du-Rhône, Atlas de l'habitat locatif social des Bouches-du-Rhône, fascicule I: Marseille, Marseille, DDE 13, 1988.

48. Sanmarco (P.), Morel (B.), Marseille : l'état du futur, Aix-en-Provence, Edisud, 1988.

49. Entretien avec un conseiller municipal PS de Marseille. 
collègues font exactement les mêmes démarches pour les électeurs qui viennent les solliciter. Les chances d'obtenir des résultats suite à une démarche personnelle sont donc très souvent pratiquement nulles. Ainsi - et cela de plus en plus à partir de la fin des années 1970 et du début des années 1980 - les interventions personnelles des élus commencent à revêtir une valeur essentiellement symbolique. Un député marseillais nous révèle en ce sens la véritable nature de la réponse qu'un élu doit aux électeurs qui le sollicitent :

«Il y a une action, je crois, avant tout un peu morale, c'est-à-dire écouter la personne, partager son souci, partager sa situation, essayer de lui dire que: "Mais oui, on peut..." Je crois que ce réconfort moral est quelque chose déjà de très important. Quant à lui garantir l'emploi, je le répète, je ne peux le faire, mais par contre ce que je lui promets, c'est de l'aider. Je lui demande s'il a déjà établi des demandes, à quel endroit, s'il s'est présenté à des concours, je me fais tenir de sa part un curriculum vitae. En fonction des endroits où il est intervenu, j'ai quelques possibilités de faire des recommandations pour qu'éventuellement sa candidature puisse être consultée de façon assez forte à l'endroit où il a adressé sa demande ; et tout cela, naturellement, ne reste pas lettre morte de ma part, puisque je tiens personnellement à informer par courrier l'intéressé des actions que je mène. C'est vrai, malheureusement, l'aboutissement dans ces domaineslà, est très limité, mais je ressens chez ces gens-là, [...] je ne dis pas des remerciements puisque je ne l'attends pas, c'est mon travail, mais qu'ils se rendent compte que $j$ 'essaye de leur venir en aide ${ }^{50}$. "

Les dossiers d'archives marseillaises que nous avons traités font comprendre que dès les années 1970-1980, une très grande quantité de lettres de recommandation ont comme seul objectif de montrer symboliquement à l'électeur que l'on s'est occupé de lui. Dans les "dossiers particuliers » de Robert Vigouroux ${ }^{51}$, à l'époque où il était adjoint de Defferre, on retrouve des séries de lettres de recommandation qui revêtent uniquement un caractère routinier et systématique, et qui sont très probablement vouées à rester sans effets. En ce qui concerne les dossiers personnels en matière de demandes d'emplois, il apparaît clairement que, suite aux courriers de demandes d'embauche reçus (à la mairie ou dans les hôpitaux de Marseille), Vigouroux adresse aux services compétents une demande générique avec une formule d'appui très vague et générique qui n'a ainsi aucune chance d'aboutir. Un exemple assez classique est celui des échanges routiniers de lettres entre Vigouroux et le directeur de l'Assistance publique au sujet des candidatures aux emplois dans les hôpitaux :

50. Entretien avec une députée PS de Marseille.

51. Archives municipales de Marseille, série 803W101-119, dossiers particuliers - Robert Vigouroux adjoint, 1983-1986. Les « dossiers particuliers " sont des dossiers nominatifs dans lesquels sont recueillies toutes les requêtes des électeurs qui, à différents titres, demandent des faveurs et des services aux adjoints de la municipalité Defferre ou au cabinet du maire. 
A une lettre de R. Vigouroux se terminant par " Je serais heureux s'il vous était possible de réserver une suite favorable à sa demande ", Monsieur M., directeur de l'Assistance publique des hôpitaux de Marseille répond ainsi : "J'ai l'honneur de vous faire connaître que ces postes sont pourvus après concours sur épreuves faisant l'objet d'une publicité par voie de presse et radiodiffusion. Aucun concours de cet ordre n'est prévu actuellement ». R. Vigouroux réécrit à l'intéressée en joignant la réponse de Monsieur M. : « Suite à mon intervention en faveur de votre demande d'emploi, je vous adresse, ci-joint, photocopie de la lettre que je viens de recevoir de Monsieur M. [...]. Vous en souhaitant bonne réception et toujours à votre disposition ${ }^{52}$. "

R. Vigouroux n'est sans doute pas sans savoir que, d'une part, on n'embauche pratiquement plus de manière directe puisque que tout le recrutement se fait désormais par concours et qu'à ce moment-là il n'y a aucun concours en vue.

Un conseiller municipal socialiste nous parle de l'existence de ce système généralisé de " fausses lettres " de recommandation:

«C'est des vraies lettres, mais on sait que c'est des fausses lettres. Je vous explique : vous venez me voir, vous me dites : "Je veux travailler à la Ville." "Il n'y a pas de problème, je vais demander au maire." J'écris au maire, je dis: "Monsieur le maire, $j$ 'ai rencontré monsieur Mattina, il veut travailler, est-ce que vous pouvez... machin, etc." Cette lettre, je vais vous la faire adresser. Vous la recevez et vous dites: "Tiens, il s'est occupé de moi." Ensuite, quinze jours après, vous recevez une lettre de la mairie de Marseille et je la reçois moi aussi, en disant : "Monsieur M. a attiré mon attention, machin, etc. Je transmets - dit le maire - au service compétent." Un mois après, le service compétent vous écrit : "J'ai reçu une lettre, Jean-Claude Gaudin a attiré mon attention sur machin, j'ai le regret de vous dire..." Voilà. Moi, je sais comment ça se passe ${ }^{53}$. "

Depuis la fin des années 1970, on voit apparaître de plus en plus des démarches de ce type. Se fait jour un véritable jeu de rôles impliquant plusieurs acteurs : l'électeur qui va voir l'élu ou lui écrit dans l'espoir d'obtenir un emploi; l'élu qui lui fait une lettre de recommandation générique et l'envoie au décideur en lui demandant surtout de répondre à l'intéressé ; le décideur (le maire ou son cabinet en l'occurrence) qui ne peut ou ne veut procéder à l'embauche et qui, selon le langage administratif, "accuse réception » en envoyant deux courriers de réponse (évidemment négative) à l'élu et à l'électeur. La réponse, même négative, des établissements ou des entreprises est très importante puisqu'elle permet de témoigner à l'électeur que, malgré le refus, on s'est bien occupé de son cas. Ce système des « fausses lettres » est d'ailleurs répandu à Marseille à tous les niveaux dans les parcours de recommandation et d'appui politique. Le cabinet du maire utilise la même

52. Ibid.

53. Entretien avec un conseiller régional PS de Marseille. 
stratégie avec des employés municipaux, des personnalités en vue, des conseillers municipaux, des militants qui sollicitent sans cesse toutes sortes de faveurs et de passe-droits. Il s'agit d'un jeu qui, dans la très grande majorité des cas, n'amène à aucun résultat concret. Il possède cependant une signification symbolique qu'explique la nécessité de maintenir chez les citoyens un certain niveau d'espoir et d'attente quant au rôle et aux possibilités qu'ont les élus de répondre à leurs demandes.

Le rétrécissement des ressources clientélaires traditionnelles finit par modifier la capacité de réponse de l'élu face à la demande privée et personnelle. Le rôle et la fonction de notable - et ainsi les processus de notabilisation des élus - se trouvent donc de plus en plus mis à mal. Le manque d'efficacité de ses interventions, sa difficulté croissante à résoudre les problèmes privés et personnels de la population lui enlèvent une certaine aura et affectent une dimension fondamentale de son activité et son rôle social : sa capacité de médiation entendue comme faculté de remplir les vides de communication existant entre différents segments et secteurs de la société54. Le notable se trouve de plus en plus en situation d'exercer un rôle de médiation qui n'est que symbolique. Il arrive à mettre en relation des acteurs sociaux en vue de favoriser des relations coopératives (ou de gérer des relations conflictuelles) dans la société urbaine, mais sa capacité directe de résoudre des problèmes et redistribuer des ressources en est de plus en plus affectée. La notabilité, dont les pratiques clientélaires et la capacité de distribuer des ressources constituent deux aspects fondamentaux, est donc remise fortement en discussion.

Une certaine tendance des sciences sociales à la catégorisation a parfois donné lieu à des lectures partielles et insatisfaisantes du phénomène notabiliaire. Selon une interprétation durcie de la pensée wébérienne, certains analystes ont considéré que l'évolution des systèmes politiques étaient caractérisée par le passage de la domination des notables à celle des professionnels de la politique, du clientélisme notabiliaire au clientélisme de parti, de la figure du notable à celle des « élus managers 55 ». La littérature italienne sur le personnel politique méridional de la Démocratie chrétienne illustre parfaitement cette conception évolutionniste. Elle s'appuie pour l'essentiel sur l'idée d'un déclin

54. Gribaudi (G.), Mediatori. Antropologia del potere democristiano nel Mezzogiorno, Turin, Rosenberg-Sellier, 1980.

55. Faure (A.), « Pouvoir local en France : le management mayoral à l'assaut du clientélisme », Politiques et management public, 9 (3), 1991. 
des notables, à partir des années 1950 , concomitant à la montée en puissance des classes moyennes dans les postes à responsabilité au sein du parti. Dans cette transformation, on a mis en avant le déclin de la clientèle personnelle des notables et la croissance du rôle des professionnels de la politique (funzionari di partito) et des appareils ${ }^{56}$. Le notable méridional de type traditionnel connu et respecté par la population aurait ainsi cédé la place à une nouvelle figure de leader local qui fonde son pouvoir sur sa position dans le parti et sur son rôle de médiateur entre le centre étatique et la périphérie. Socialement, il s'agirait de moins en moins de rentiers, de propriétaires terriens, de professions libérales et de plus en plus d'une classe moyenne composée de fonctionnaires publics et d'enseignants ${ }^{57}$.

Ces modèles binaires nous apparaissent critiquables car ils négligent, au sein des processus historiques, la pluralité d'éléments de continuité et de discontinuité en ce qui concerne l'origine sociale des élus, leurs pratiques et les ressources qu'ils arrivent à mobiliser. En étudiant les pratiques clientélaires des élus marseillais et les ressources qu'ils ont été susceptibles de redistribuer, nous avons vu que la notabilité se transforme, se modifie selon les personnes, leurs pratiques et les contextes territoriaux et historiques dans lesquels ils agissent. Les processus de notabilisation ne dépendent pas uniquement du statut social et du prestige accumulé par héritage familial et historique. La notabilité politique dépend aussi de la capacité d'un élu à se faire une place dans des structures partisanes locales et à savoir accéder aux niveaux institutionnels de décision lui permettant de contrôler la redistribution clientélaire des ressources. Les pratiques clientélaires, attribut traditionnel des notables, ne disparaissent donc pas avec la professionnalisation de la politique: elles deviennent partie intégrante du métier d'élu. A Marseille, elles ont été intégrées à l'intérieur de l'administration municipale qui les a souvent codifiées et légitimées. Les techniciens du cabinet du maire et des services de la mairie participaient eux-mêmes à la codification de règles officieuses favorisant par exemple les enfants du personnel de la mairie dans l'accès aux emplois publics. Le système d'affectation des logements sociaux par groupe politique a participé de la même logique clientélaire érigée en système à l'intérieur de l'administration.

56. Cf. Minolfi (S.), Vigilante (R.), "Il ceto politico locale in Campania in età repubblicana ", Italia contemporanea, 167, 1987

57. Minolfi (S.), Soverina (F.), L'incerta frontiera. Saggio sui consiglieri comunali a Napoli. 1946-1992, Naples, Edizioni scientifiche italiane, 1993. 